\title{
A AMOSTRAGEM EM SNOWBALL (BOLA DE NEVE) EM UMA PESQUISA QUALITATIVA NO CAMPO DA ADMINISTRAÇÃO
}

\author{
Beatriz Rodrigues Silva Bockorni ${ }^{1}$ \\ Almiralva Ferraz Gomes ${ }^{2}$
}

BOCKORNI, B. R. S.; GOMES, A. F. A amostragem em snowball (bola de neve) em uma pesquisa qualitativa no campo da administração. Revista de Ciências Empresariais da UNIPAR, Umuarama, v. 22, n. 1, p. 105-117, jan./jun. 2021.

RESUMO: A amostra em bola de neve, ou snowball, é uma técnica de amostragem que se utiliza de redes de referência, por isso, torna-se apropriada para pesquisas com grupos de difícil acesso ou até mesmo quando se trata de temas mais privados. O presente artigo objetivou discutir a adoção e o uso da amostragem em snowball em uma pesquisa qualitativa da área de Administração, discutindo suas limitações e vantagens. Para tal, foram utilizadas como referências Albuquerque (2009), Biernacki e Waldorf (1981), Baldin e Munhoz (2011). O estudo, portanto, conclui que a amostra em bola de neve é vantajosa, ao ser útil para pesquisas de cunho qualitativo. No entanto, deve-se compreender que esse tipo de amostragem não se sustenta por si só, pois deve ser aliado a outros tipos, ademais, depende da execução adequada das demais fases da pesquisa.

PALAVRAS-CHAVE: Administração; Amostragem; Bola de neve; Metodologia; Pesquisa qualitativa.

\section{SNOWBALL SAMPLING IN A QUALITATIVE BUSINESS RESEARCH}

ABSTRACT: A snowball sample is a sampling technique that uses reference networks. As such, it can be applied in research with difficulty in accessing groups or even when dealing with topics that are more private. This article aims at discussing the adoption and use of snowball sampling in qualitative research in the Business area, presenting its limitations and advantages. To this end, the works of Albuquerque (2009), Biernacki \& Waldorf (1981), Baldin \& Munhoz (2011) were used as reference. It could be concluded from the study that a snowball sample is advantageous, being useful for qualitative research. However, it must be noted that this type of sampling cannot be used by itself, but

DOI: https://doi.org/10.25110/receu.v22i1.8346

${ }^{1}$ Bacharel em Administração pela Universidade Estadual do Sudoeste da Bahia. Rua São Roque, 55 Bairro Guarani, Vitória da Conquista - Bahia. E-mail: beatrizrsbockorni@gmail.com

${ }^{2}$ Doutora em Administração pela Universidade Federal de Lavras. Professora Plena da Universidade Estadual do Sudoeste da Bahia. Rua Macário Novais Figueira, 11, Bairro Candeias, Vitória da Conquista - Bahia. E-mail: almiralva@gmail.com 
in combination with other types, and also depends on the appropriate execution of all phases in the research.

KEYWORDS: Business; Sampling; Snowball; Methodology; Qualitative research.

\section{MUESTREO EN SNOWBALL (BOLA DE NIEVE) EN UNA INVESTIGACIÓN CUALITATIVA EN EL ÁMBITO DE LA ADMINISTRACIÓN}

RESUMEN: La muestra en Bola de Nieve o Snowball, es una técnica de muestreo que se utiliza de redes de referencia, por eso, se hace apropiada para investigaciones con grupos de difícil acceso o incluso cuando se trata de temas más privados. Este artículo tuvo como objetivo discutir la adopción y uso de muestreo en Snowball, en una investigación cualitativa en el área de Administración, discutiendo sus limitaciones y ventajas. Para eso, se utilizaron como referencias Albuquerque (2009), Biernacki y Waldorf (1981), Baldin y Munhoz (2011). Por tanto, el estudio concluye que la muestra en Bola de Nieve es ventajosa, ya que es útil para la investigación cualitativa. Sin embargo, debe entenderse que ese tipo de muestreo no se sostiene por sí mismo, ya que debe ser aliado a otros tipos, además, depende de la correcta ejecución de las otras fases de la investigación.

PALABRAS CLAVE: Administración; Muestreo; Bola de Nieve; Metodología; Investigación cualitativa.

\section{INTRODUÇÃO}

A amostra em snowball, ou bola de neve, é uma técnica de amostragem que vem sendo utilizada em pesquisas qualitativas, nos últimos anos, principalmente, porque permite que se alcancem populações pouco conhecidas ou de difícil acesso. Em outras palavras, a amostra do tipo bola de neve destacase em pesquisas que optam por amostras não probabilísticas em estudos de natureza qualitativa. As pesquisas qualitativas, segundo Dyniewicz (2009), são baseadas na premissa de que o conhecimento sobre as pessoas só é possível pela descrição das experiências humanas, tais como elas são vividas e definidas pelos seus próprios atores. Malhotra (2011) ainda defende que a pesquisa qualitativa é adequada também para situações de incerteza, quando os resultados conclusivos diferem das expectativas no momento do planejamento da pesquisa, pois é capaz de fornecer clareza ao aspecto a ser pesquisado. Dessa forma, a técnica de bola de neve se mostra apropriada para pesquisas desse tipo.

O tipo bola de neve, portanto, é uma forma de amostra não probabilística 
que usa redes de referência e indicações. Ademais, é bastante útil para pesquisar grupos difíceis de serem estudados ou acessados ou também quando não se conhece o universo da pesquisa. É uma técnica útil ainda para se estudar questões delicadas que são de âmbito privado e requerem o conhecimento de pessoas já pertencentes aos grupos para se localizar informantes. No entanto, essa forma de amostragem não é capaz de se sustentar sozinha, por isso, faz-se necessário que se preste atenção às sutilezas do campo pesquisado (VINUTO, 2014).

A amostra em bola de neve possui vantagens e desvantagens que devem ser levadas em consideração ao se optar por esse tipo de amostragem. Dessa forma, o presente artigo objetiva discutir a adoção e o uso da amostragem em snowball em pesquisas qualitativas da área de Administração, discutindo suas limitações e vantagens. Para tanto, trará a experiência de um estudo acerca da percepção de sucesso e fracasso de empreendedores de uma cidade de porte médio, localizada no interior da Bahia. O tema da pesquisa propiciou o uso da amostragem em bola de neve, pois permitiu que os próprios indivíduos entrevistados indicassem outros que, com a corroboração de seus pares, aceitavam comentar sobre temas ainda considerados como tabus por alguns deles, tal como o fracasso de um empreendimento ou as dificuldades encontradas no processo de gestão. Tal investigação realizou entrevistas com nove empreendedores que relataram suas experiências e definições sobre sucesso e fracasso, assim como suas histórias acerca da trajetória de vida e provável relação com as definições atribuídas ao sucesso e ao fracasso.

$\mathrm{O}$ artigo foi organizado em quatro partes. A primeira trata-se dessa introdução. A segunda aborda o marco teórico adotado, indicando algumas definições, vantagens e limitações da técnica de bola de neve. A terceira parte traz um exemplo prático de uma pesquisa de natureza empírica em que a técnica de bola de neve foi utilizada para a obtenção da amostra. E, por fim, a quarta parte apresenta as considerações finais deste artigo.

\section{MARCO TEÓRICO}

A amostragem em snowball ou bola de neve pode ser definida como um tipo de amostragem não probabilística, em que se utilizam cadeias de referência, além disso, é útil em pesquisas com grupos de difícil acesso (VINUTO, 2014). Segundo Dewes (2013), os grupos de difícil acesso podem ser definidos como membros de uma população que não são distinguíveis na população geral, ou cujo comportamento envolve um tema de cunho sensível que faz com que eles não desejem se revelar, sejam por questões de ilegalidade ou por que são reprovados socialmente ou simplesmente por que não desejam se expor.

Lindstrom (2016) afirma que a técnica de bola de neve se mostrou efetiva 
para encontrar membros de grupos de difícil acesso, em especial em estudos que envolvem imigrantes, cuja minoria na população de destino pode tornar o acesso a eles difícil, principalmente se estiverem em situação de documentação irregular, o que, na maioria das vezes, explica os motivos pelos quais não querer ser encontrados ou mesmo identificados.

Para a realização de uma amostragem em bola de neve é necessário que haja um intermediário inicial, também denominado de semente, que localiza ou aponta algumas pessoas com o perfil necessário para a pesquisa a ser realizada. A semente pode, inclusive, ser um dos participantes. As pessoas indicadas são então solicitadas a indicarem mais pessoas. Dessa forma, a amostra, que antes era difícil de ser calculada probabilisticamente ou até mesmo de difícil acesso, expande-se, na maioria das vezes, satisfatoriamente. Segundo Baldin e Munhoz (2011, p. 5),

A snowball sampling ou "Bola de Neve" prevê que o passo subsequente às indicações dos primeiros participantes no estudo é solicitar, a esses indicados, informações acerca de outros membros da população de interesse para a pesquisa (e agora indicados por eles), para, só então sair a campo para também recrutá-los.

Sobre as sementes, Albuquerque (2009) afirma que podem ser recrutadas tanto pelos próprios pesquisadores, quanto pelos indivíduos que potencialmente serão pesquisados ou por sujeitos que possuem um conhecimento da comunidade/ fenômeno a ser investigado. Esse momento, também denominado de onda zero, segue-se até que haja saturação na amostra (DEWES, 2013; VINUTO, 2014). A World Health Association (1994) define que o ponto de saturação se dá com o alcance dos objetivos da pesquisa ou quando os novos nomes não tragam informações novas ou relevantes ao estudo.

Goodman (1961) afirma que os estágios podem ser vistos como "s estágio k" ( $s$ stage $k$ ), em que uma quantidade aleatória de indivíduos é escolhida dentro de uma população alvo e questionada acerca de outros indivíduos $k$, que podem ser relacionados com estes por diversos fatores, tais como a amizade ou relações familiares. Esses indivíduos mencionados irão compor o primeiro estágio e então devem informar outros que formarão o estágio dois e assim por diante. Desse modo, a técnica de bola de neve pode ser definida como uma técnica de amostragem que utiliza de cadeias de referência, como uma rede para coleta de informações, usando técnicas de recrutamento em cadeia (BALDIN; MUNHOZ, 2011).

No entanto, esse tipo de amostragem possui limitações quanto a sua escolha, visto que não é apropriada para pesquisas relacionadas à probabilidade 
ou em que haja a necessidade de se conhecer com precisão o total do universo a ser estudado. Portanto, é melhor utilizada em pesquisas em que não há a necessidade de se conhecer a quantidade da população e quando a pesquisa pode estar relacionada à questões problemáticas ou privadas, em que os indivíduos da população têm altas chances de se conhecerem, ou de terem ligações entre si (BIERNACKI; WALDORF, 1981; DEWES, 2013; VINUTO, 2014). Quanto às limitações da pesquisa com amostragem em bola de neve, Vinuto (2014) considera que se a pesquisa for relacionada a uma população de pequeno porte, em que todos têm contato uns com os outros, a amostragem em snowball pode chegar até uma amostragem exaustiva, o que, por sua vez, compreende em duas fases até chegar a uma amostra representativa da população total.

No caso de uma população maior, dificilmente se chegará a uma amostra representativa e aleatória, visto que as pessoas mais "populares", ou conhecidas por todos dentro de um grupo, irão ser mais indicadas. No entanto, salienta-se que em populações maiores, há mais chances de os entrevistados conhecerem mais pessoas, se comparado com uma população mais reduzida. Esse parece ser um motivo para que a amostragem em bola de neve não seja tão eficaz com populações de grandes dimensões, pois nem todos os indivíduos do universo a ser investigado terão a mesma chance de serem indicados aleatoriamente pelas sementes, visto que há menores chances de todos se conhecerem (VINUTO, 2014).

Dessa forma, compreende-se que a amostragem em bola de neve é mais indicada para pesquisas com grupos menores ou, em caso de grupos maiores, em que haja a possibilidade de encontrar diversas sementes, que elas sejam capazes de indicar membros em seu círculo social de diversos níveis de "popularidade" ou até mesmo sementes com contatos mais diversificados dentro do grupo. Essas estratégias podem levar a uma amostra mais representativa do grupo social a ser pesquisado.

Um dos inconvenientes dessa forma de pesquisa é a possibilidade da maior parte das argumentações serem semelhantes, em caso da amostra ser composta por pessoas do mesmo círculo social. Outra desvantagem, também associada ao círculo social, é que a maior parte das pessoas acessadas são as mais visíveis na população. No entanto, isso pode ser evitado ao se obter sementes de redes sociais diversas (VINUTO, 2014; ALBUQUERQUE, 2009). O acesso de pessoas de maior visibilidade pode não ser considerado uma desvantagem, dependendo do tipo de população a ser acessada e o tipo de pesquisa a ser realizada.

Lindstrom (2016) afirma que outra desvantagem da técnica em bola de neve é a possibilidade de ocorrer um viés na amostra quanto à estimação do total da população, que pode ser subestimada ou superestimada, visto que são 
amostras não probabilísticas.

As vantagens desse tipo de pesquisa incluem seu custo, visto que não necessita de tantos recursos como outros tipos de amostragem de grupos de difícil acesso, ao envolver uma relação de confiança que auxilia a amostragem e facilitar que os informantes aceitem participar da pesquisa, visto que já seriam indicados por alguém conhecido (DEWES, 2013). As vantagens e desvantagens da amostragem por bola de neve, destacadas no Quadro 1, devem ser verificadas antes da sua escolha como método para a pesquisa, já que podem gerar empecilhos dependendo do estudo a ser realizado.

Quadro 1: Vantagens e desvantagens da amostragem em bola de neve

\begin{tabular}{|l|l|}
\hline \multicolumn{1}{|c|}{ Vantagens } & \multicolumn{1}{c|}{ Desvantagens } \\
\hline -É ideal para pesquisas sobre temas de & -Não é apropriada para pesquisas \\
âmbito privado ou com populações de & probabilísticas. \\
difícil acesso. & -Não há como conhecer o total da \\
-Os indivíduos são mais propensos & população pesquisada. \\
a aceitarem participar, por serem & -Há o risco de obter sempre as mesmas \\
indicados por conhecidos. & respostas. \\
-Há acesso às pessoas de maior & -O pesquisador pode receber sempre \\
visibilidade dentro do grupo social & as mesmas indicações. \\
pesquisado. & -Há a possibilidade de ocorrer um \\
-Baixo custo. & viés amostral, por superestimação ou \\
& subestimação da amostra. \\
\hline
\end{tabular}

Fonte: Elaboração própria, com base nos estudos de Biernacki e Waldorf (1981), Dewes (2013) Vinuto (2014), Albuquerque (2009), Lindstrom (2016) e Dewes, 2013.

Um tipo semelhante de amostragem não probabilística é a amostra orientada pelo respondente (respondent-driven sampling). Aliás, a similaridade entre elas pode gerar confusão. No entanto, na amostra orientada pelo respondente é crucial que se obtenham estimativas da quantidade de pessoas no círculo social do respondente, a fim de se estimar a população, assim como obter informações definidas sobre quem recrutou quem. Outra diferença reside no fato de a amostra orientada pelo respondente prover de um sistema duplo de incentivo aos respondentes, que recebem um incentivo monetário ao participarem da pesquisa e outro por cada pessoa indicada que também participa. De acordo com esse modelo de amostragem, semelhantemente ao modelo em bola de neve, os participantes são mais propensos a participar ao serem indicados e motivados por seus amigos, em especial ao lidar com temas sensíveis e complexos (SCHONLAU; LIEBAU, 2012).

Como afirma Vinuto (2014, p. 217), uma amostragem em bola de 
neve "é apenas uma ferramenta, num contexto maior de trabalho, que pode auxiliar o pesquisador, mas que depende necessariamente da boa execução das outras fases da pesquisa". Ou seja, seu funcionamento satisfatório depende ainda da consideração de outros meios e da adequada execução de todas as fases, principalmente das fases iniciais, como a onda zero. Em outros termos, ela ainda depende de outros meios e da execução das outras fases, que devem ser adequadamente planejadas e desenvolvidas, a fim de se manter a rede de contatos que constituirá a amostra da pesquisa. Contudo, mostra-se proveitosa para pesquisas qualitativas, em que se busca investigar mais acerca de uma população ainda pouco conhecida ou explorada, auxiliando no entendimento geral de fatores associados a essa população ou ao seu modo de vida em geral.

\section{A ADOÇÃO DA TÉCNICA DE BOLA DE NEVE (SNOWBALL) EM UMA PESQUISA QUALITATIVA NO CAMPO DA ADMINISTRAÇÃO}

A pesquisa aqui exemplificada foi o resultado de uma investigação que tinha como objetivo analisar a percepção de sucesso e fracasso de empreendedores de um município de porte médio, localizada no interior da Bahia. Porém, não se conhecia o universo da pesquisa, pois não havia nenhum tipo de levantamento local que indicasse a quantidade de sujeitos que haviam experimentados o sucesso e/ou fracasso nesse município. Ademais, tratar do tema fracasso é constrangedor para muitos que o experimentam e tendo em vista que, segundo Dewes (2013), o snowball também se aplica a indivíduos ou grupos que espontaneamente não desejam se expor, tal técnica se revelou como apropriada para se chegar aos sujeitos da pesquisa. Além disso, esse tipo de amostragem se apresentou como uma alternativa promissora para se chegar a um número satisfatório de sujeitos, logo que se tinha conhecimento e acesso a alguns indivíduos que se enquadravam no perfil desejado para o estudo, ou seja, de homens ou mulheres que haviam vivenciado sucesso e/ou fracasso empresarial,

Durante a pesquisa buscou-se, intencionalmente, investigar casos de sucesso e de fracasso empresarial no município. Os intermediários, ou sementes, foram selecionados por seu conhecimento sobre a população a ser pesquisada. Desse modo, além de se buscar, na onda zero, membros da população a ser investigada, tendo em vista a proposição de Albuquerque (2009) de que as sementes tão podem ser compostas por sujeitos que não necessariamente vivenciaram o fenômeno estudado, mas que têm conhecimento a respeito dele recorreu-se a professores do curso de Administração de uma universidade pública que oferece o referido curso há mais de três décadas, muito embora eles não tivessem passado por tal experiência. Assim, como tais sementes conheciam a comunidade local indicaram empresários(as) que já haviam experimentado 
sucesso e/ou fracasso.

Os contatos com as sementes e com os empreendedores foram realizados por meio dos mais diversos caminhos, a fim de se obter contato com os mais diversos grupos de empreendedores na onda zero, portanto, iniciou-se com seis intermediários e, chegou-se a uma amostra de 41 empreendedores. Desse total, nove aceitaram ser entrevistados, quatro pediram mais tempo para definir sua participação, no entanto, não houve tempo hábil para a sua entrevista durante a realização da pesquisa, e 28 se recusaram a participar (Figura 1).

Figura 1: Técnica de snowball
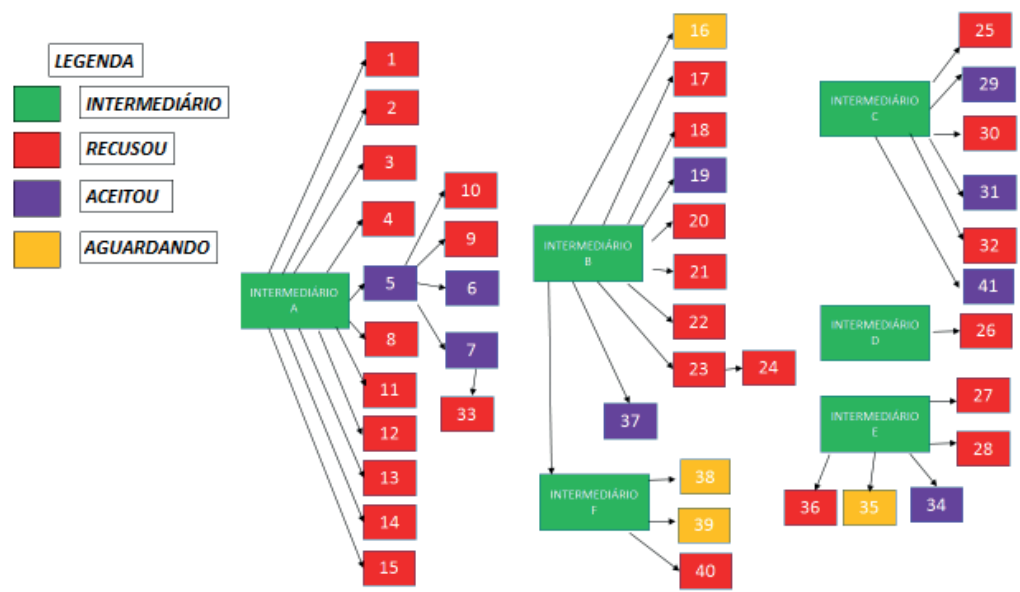

Fonte: Pesquisa de campo, 2019.

Dessa forma, a amostra final da pesquisa foi de nove empreendedores, que atuam ou atuaram nesta cidade interiorana. Desse total, cinco somente falaram de seus sucessos, apesar de alguns terem tido fracassos em empreendimentos prévios, três falaram de seus sucessos e fracassos, e um abordou somente o fracasso experimentado. A classificação quanto a sucesso ou fracasso foi definida de modo que o fracasso fosse classificado pelo encerramento das atividades do empreendedor na empresa (seja por falência, venda ou o seu afastamento), e o sucesso como a sua continuidade.

A maior parte dos entrevistados recusou participar da pesquisa logo no primeiro contato, quando foram informados acerca do tema da pesquisa e de seus objetivos, assim como do tempo médio que seria necessário para a realização da 
entrevista, que era, em média, de 30 minutos. Os empreendedores foram também informados que nem seus nomes, nem os nomes de suas empresas, seriam divulgados, sendo tratados anonimamente ao longo da pesquisa, e na divulgação de seus resultados.

Durante o período de definição da amostra, alguns empreendedores até chegaram a aceitar, porém, mais tarde, alguns cancelaram a participação e outros a adiaram por muitas vezes ao ponto de após várias tentativas de reagendamento da entrevista, decidirem pela desistência e justificarem que não tinham mais disponibilidade para tratar de assuntos relacionados às empresas fechadas. A recusa em participar pode ser atribuída, na maior parte dos casos, à dificuldade dos sujeitos em abordar temas como o fracasso pessoal, considerado ainda por muitas pessoas como um tabu, além do tempo que necessitariam dispor para a pesquisa.

Os entrevistados que aceitaram participar da pesquisa tiveram ressalvas acerca das respostas sobre fracasso, limitando-se ou recusando-se a responder as questões pertinentes ao tema, ou até mesmo atendo-se, em muitos casos, a mera afirmação de que fracasso se tratava do oposto do sucesso. Por fim, os entrevistados foram solicitados a indicar um ou mais empreendedores que já passaram por experiências de sucesso ou fracasso, a fim de compor a amostra da pesquisa, no entanto, a maior parte não indicava nenhum sujeito, principalmente quando eram solicitados a indicar pessoas que haviam experimentado o fracasso, presumidamente por medo de indicarem amigos ou colegas que se sentiriam ofendidos ao serem tratados como casos de fracasso.

A pesquisa qualitativa se apresenta como a mais apropriada quando se pretende investigar fenômenos pouco estudados e que dependem da compreensão dos agentes a serem investigados sobre o que os levou a tomar as decisões que tomaram: "Essa empreitada só é possível se os sujeitos forem ouvidos a partir da sua lógica e exposição de razões" (GODOI; BALSINI, 2006, p. 91). Em outras palavras, a pesquisa qualitativa visa se integrar ao seu objeto de estudo, para assim compreendê-lo da melhor forma possível (TOLEDO; GONZAGA; BARBOSA, 2011). Dessa forma, utiliza informações descritivas que the auxiliam na interpretação dos dados coletados. Gomes (2002) afirma que, apesar da utilidade da metodologia, o pesquisador pode ter dificuldades na articulação de conclusões que surjam de dados concretos com conhecimentos mais amplos ou subjetivos. Gomes e Santana (2010) corroboram, ao afirmarem que essa dificuldade pode ser percebida em alguns fatores, tais como a vulnerabilidade da pesquisa qualitativa no que tange a ocultação de informações por parte dos respondentes e a manipulação de que os sujeitos investigados podem exercer, visto que o pesquisador pode vir a ser manipulado ou influenciado a pensar e ver as situações de acordo com a forma que os entrevistados as veem. No 
entanto, compreende-se que a análise qualitativa pode garantir uma maior compreensão dos dados coletados, garantindo um aprofundamento no tema a ser pesquisado, assim como facilitando a coleta de dados acerca de assuntos difíceis de serem quantificados, como as percepções e apegos emocionais dos indivíduos (MALHOTRA, 2011).

A amostra em snowball foi utilizada nesta pesquisa ao se adotar uma amostragem em cadeia de referência, que permitiu, por sua vez, o acesso a uma população que, apesar de conhecida e ser considerada de fácil acesso quanto à identificação de casos de sucesso, era de difícil acesso em se tratando de experiências de empreendedores que experimentaram o fracasso, afinal de contas, pressupunha-se que tratar dos fracassos seja uma decisão difícil para a maioria das pessoas, pois, de um modo geral, deseja-se afastá-los da memória e enterrálos, preferencialmente, no passado, e não trazê-los para o presente, o que pode trazer dor e sofrimento que nem todos estão dispostos a revivê-los e reverenciálos. Desse modo, as indicações para futuras participações foram limitadas a casos de sucesso, pois os participantes não se sentiram à vontade de indicar indivíduos que experimentaram o fracasso com receio de que os indivíduos apontados como não bem sucedidos se ofendessem.

Dessa forma, compreende-se que a técnica de snowball foi escolhida e utilizada e útil nesta pesquisa devido às suas características para amostragem de uma população de difícil acesso e que não é aberta a questionamentos sobre temas considerados tabus, como o fracasso. No entanto, a técnica de snowball ainda é pouco difundida nas ciências sociais aplicadas. Ademais, nem todos os participantes aceitaram se tornar também intermediários, o que culminou na diminuição da amostra final da pesquisa, muito embora não tenha comprometido o alcance do objetivo proposto.

\section{CONSIDERAÇÕES FINAIS}

A amostra em snowball, apesar de vantajosa em pesquisas em que não se conhece a população a ser pesquisada ou em se tratando de perguntas mais privadas ou polemizadas, ainda não é sustentável por si só, pois depende de outros meios de pesquisa e da execução das outras fases da pesquisa a ser realizada, por isso, ainda é pouco adotada.

A pesquisa representada abordava casos de sucesso e fracasso empresarial em um município do interior baiano e tinha como objetivo analisar a percepção de sucesso e fracasso de empreendedores e relacioná-la às trajetórias de vida dos sujeitos estudados. O referido estudo concluiu que o sucesso e o fracasso são só estão associados às realizações pessoais e profissionais como também ligados a fatores econômicos, tais como o lucro. Ademais, as maiores 
dificuldades enfrentadas pelos empreendedores investigados se encontraram nos conflitos interpessoais. Os motivos para abertura das empresas foram, em sua maioria, relacionados à necessidade, à oportunidade e a influência familiar. Os motivos para encerramento das empresas, por sua vez, foram associados a dois fatores principais, a falta de conhecimento sobre o negócio e problemas financeiros.

Na pesquisa exemplificada, a amostragem em snowball foi vantajosa ao facilitar o acesso aos indivíduos, de forma que uns poderiam indicar aos outros, gerando mais confiança dos entrevistados para com a pesquisa. Salienta-se que, apesar da existência de diversos dados acerca de empresas de sucesso assim como de empresas que experenciaram fracassos, os próprios empreendedores ainda não se sentem confortáveis para discutir especialmente sobre os fracassos sofridos, o que justifica a dificuldade de os indivíduos entrevistados discorrer sobre o tema e, por sua vez, indicar outros participantes, além de manifestar certa indisponibilidade de tratar de um tema com implicações pessoais e profissionais. Apesar disso, sem a amostragem em snowball a pesquisa poderia sofrer impactos quanto à quantidade de empreendedores a serem entrevistados, visto que foram as sementes iniciais que auxiliaram na obtenção de maiores contatos abertos a tratar sobre o tema.

Sendo assim, a amostragem em bola de neve se mostra como uma estratégia de pesquisa de grande utilidade nos estudos qualitativos ao viabilizar mais facilmente a obtenção de uma amostra. Contudo, é necessário considerar que esse tipo de amostragem demanda a compreensão do campo a ser pesquisado, assim como das suas nuances.

Entre as limitações presentes nesta pesquisa se encontra a escassez de literatura que trata do assunto, assim como a baixa utilização da metodologia pelo país, que acaba limitando os estudos sobre a amostragem do tipo bola de neve. Desse modo, recomendam-se maiores pesquisas acerca do tema, a fim de não só se expandir sua conceituação e utilização, assim como também produzir pesquisas sobre o seu estado-da-arte no Brasil.

\section{REFERÊNCIAS}

ALBUQUERQUE, Elisabeth Maciel de. Avaliação da técnica de amostragem "Respondent-driven Sampling” na estimação de prevalências de Doenças Transmissíveis em populações organizadas em redes complexas. 2009. $99 \mathrm{f}$. Dissertação (Mestrado em Saúde) - Escola Nacional de Saúde Pública Sérgio Arouca Fiocruz, Rio de Janeiro, 2009. Disponível em: https://www.arca.fiocruz. br/handle/icict/2411. Acesso em: 21 jan. 2020 
BALDIN, Nelma; MUNHOZ, Elzira M. B. Snowball (bola de neve): uma técnica metodológica para pesquisa em educação ambiental comunitária. In: Congresso Nacional de Educação, 10., 2011. Anais... Curitiba: PUCPR, 2011. Disponível em: https://docplayer.com.br/1714932-Snowball-bola-de-neve-umatecnica-metodologica-para-pesquisa-em-educacao-ambiental-comunitaria.html. Acesso em: 21 jan. 2020.

BIENARCKI, Patrick; WALDORF, Dan. Snowball Sampling: problems and techniques of chain referral sampling. Sociological Methods \& Research, Cambridge-Massachusetts, v. 10. n. 2, nov, 1981. p.141-163. Disponível em: https://journals.sagepub.com/doi/10.1177/004912418101000205. Acesso em: 21 jan. 2020.

DEWES, João Osvaldo. Amostragem em bola de neve e respondent-driven sampling: uma descrição dos métodos. 2013. 53f. TCC (Graduação) - Curso de Estatística, Universidade Federal do Rio Grande do Sul, Porto Alegre, RS, 2013. Disponível em: https://lume.ufrgs.br/handle/10183/93246. Acesso em: 21 jan. 2020.

\section{DYNIEWICZ, Ana Maria. Metodologia da pesquisa em saúde para} iniciantes. 2. ed. São Caetano do Sul: Difusão Editora, 2009.

GODOI, Christiane K.; BALSINI, Cristina P. V. A pesquisa qualitativa nos estudos organizacionais brasileiros: uma análise bibliométrica. In: GODOI, Christiane K.; BANDEIRA-DE-MELLO, Rodrigo; SILVA Anieleson B. da. (Orgs.). Pesquisa qualitativa em estudos organizacionais: paradigmas, estratégias e métodos. São Paulo: Saraiva, 2006.

GOMES, Almiralva Ferraz; SANTANA, Weslei Gusmão Piau. A história oral na análise organizacional: a possível e promissora conversa entre a história e a administração. Cadernos Ebape.BR, Rio de Janeiro, v. 8, n. 1, mar. 2010. Disponível em: http://www.scielo.br/scielo.php?pid=S167939512010000100002\&script=sci_abstract\&tlng=pt. Acesso em: 23 mar. 2020

GOMES, Romeu. A análise de dados em pesquisa qualitativa. In: DESLANDES, Suely Ferreira; CRUZ NETO, Otavio; GOMES, Romeu; MINAYO, Maria Cecília de Souza. (Orgs.) Pesquisa Social: teoria, método e criatividade. 21 ed. Petrópolis: Vozes, 2002. Disponível em: https://wp.ufpel. edu.br/franciscovargas/files/2012/11/pesquisa-social.pdf. Acesso em: 21 abr. 2020 . 
GOODMAN, Leo. Snowball Sampling. The Annals of Mathematical Statistics, Beachwood-Ohio, v. 32, n. 1. p.148-170, 1961. Disponível em: https://www.jstor.org/stable/2237615. Acesso em: 15 abr. 2020.

LINDSTROM, David. How representative are snowball samples? Using the Ethnosurvey to study Guatemala-U.S. migration. The Annals of The American Academy of Political and Social Science, Philadelphia-USA, v. 666. p.64-76, 2016. Disponível em: https://www.jstor.org/stable/24756128?seq=1\#metadata info_tab_contents. Acesso em: 13 abr. 2020.

MALHOTRA, Naresh. Pesquisa de marketing: foco na decisão. São Paulo: Pearson Prentice Hall, 2011. Disponível em: https://www.academia. edu/19104649. Acesso em: 24 abr. 2020.

SCHONLAU, Matthias; LIEBAU, Elisabeth. Respondent-driven sampling. The Stata Journal, Texas-USA, v. 12, n. 1. p.72-93, 2012. Disponível em: https:// journals.sagepub.com/doi/10.1177/1536867X1201200106. Acesso em: 13 abr. 2020 .

TOLEDO, Cézar de Alencar Arnaut; GONZAGA, Maria Tereza Claro; BARBOZA, Marcos Ayres. A ética na pesquisa acadêmica envolvendo seres humanos. In: TOLEDO, Cézar de Alencar Arnaut; GONZAGA, Maria Tereza Claro (Orgs.). Metodologia e técnicas de pesquisa nas áreas de ciências humanas. Maringá: Eduem, 2011. Disponível em: http://old.periodicos.uem. br/ eduem/novapagina/?q=system/files/Liv-Cezar_1a.pdf. Acesso em: 22 abr. 2020 .

VINUTO, Juliana. A amostragem em bola de neve na pesquisa qualitativa: um debate em aberto. Temáticas, Campinas, v. 22, n. 44, p. 203-220, 2014. Disponível em: https://www.academia.edu/16320788/A_Amostragem_em_ Bola_de_Neve_na_pesquisa_qualitativa_um_debate_em_aberto. Acesso em: 21 jan. 2020 .

WORLD HEALTH ASSOCIATION. Division of Mental Health. Qualitative Research for Health Programmes. Geneva: WHA, 1994. Disponível em: https:// apps.who.int/iris/handle/10665/62315. Acesso em: 21 jan. 2020.

Recebido em: $15 / 12 / 2020$

Aceito em: 05/05/2021 